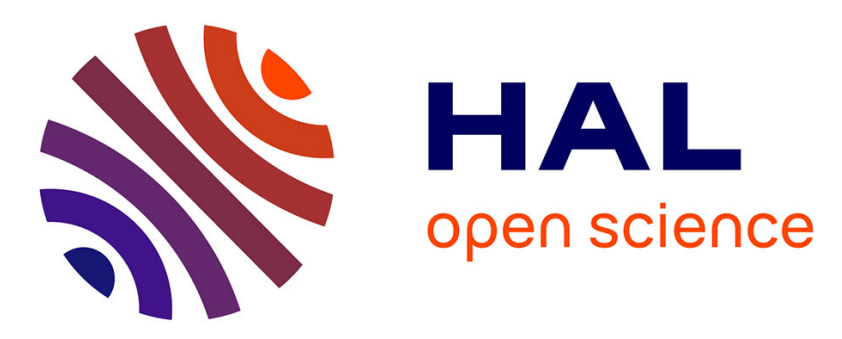

\title{
A block replacement policy for a bivariate wear subordinator
}

\author{
Sophie Mercier, M. Roussignol
}

\section{To cite this version:}

Sophie Mercier, M. Roussignol. A block replacement policy for a bivariate wear subordinator. European Safety and Reliability Conference: Advances in Safety, Reliability and Risk Management, ESREL, 2011, Troyes, France. pp.1007-1014. hal-00868118

\section{HAL Id: hal-00868118 https://hal.inria.fr/hal-00868118}

Submitted on 19 Apr 2018

HAL is a multi-disciplinary open access archive for the deposit and dissemination of scientific research documents, whether they are published or not. The documents may come from teaching and research institutions in France or abroad, or from public or private research centers.
L'archive ouverte pluridisciplinaire HAL, est destinée au dépôt et à la diffusion de documents scientifiques de niveau recherche, publiés ou non, émanant des établissements d'enseignement et de recherche français ou étrangers, des laboratoires publics ou privés. 


\title{
A block replacement policy for a bivariate wear subordinator
}

\author{
Sophie Mercier \\ Laboratoire de Mathématiques et de leurs Applications, Université de Pau et des Pays de l'Adour, France
}

Michel Roussignol

Laboratoire d'Analyse et de Mathématiques Appliquées, Université Paris-Est Marne-la-Vallée, France

\begin{abstract}
Classical models for wear accumulative indicators are univariate Gamma and compound Poisson processes, which both are univariate subordinators (increasing Lévy processes). Bivariate subordinators are here proposed to model correlated couples of univariate wear indicators, namely bivariate wear indicators. A few properties of these bivariate subordinators are pointed out, and a special simple construction is provided, for application purpose. The use of these bivariate wear subordinors is illustrated through the study of a classical block replacement policy. The influence of the dependence between the marginal indicators on the optimal block replacement policy is pointed out.
\end{abstract}

\section{INTRODUCTION}

In case of a system submitted to an accumulative random damage, classical stochastic models are compound Poisson processes and Gamma processes, which both are increasing Lévy processes, see (Van Noortwijk 2009), (Nakagawa 2007) or (AbdelHameed 1975) e.g.. Such classical wear models typically are univariate. However, the deterioration level of a system cannot always be synthetized into one single indicator and several indicators may be necessary, see (Mercier et al. 2011) for an industrial example. In that case, a multivariate wear model must be used to account for the dependence between the different univariate indicators of the system. Another context where multivariate wear models are required is the case of different systems submitted to common stresses, which make their wear indicators dependent. Multivariate increasing stochastic models hence are of interest in different contexts.

We here propose to use multivariate increasing Lévy processes (or multivariate subordinators) as wear processes. Two main arguments are the following: first, such processes present some positive dependence property (consequence of (Bäuerle et al. 2008)); secondly, thresholds reaching times may be proved to present aging properties. Such properties are typical of what one may expect from a multivariate wear model: indeed, correlation between wear indicators reveals some common stresses or so, and high values for one of these indicators should entail higher values for the other ones. Roughly speaking, this is the positive dependence property. Also, in a reliability context, thresholds reaching times for increasing wear processes typically represent times to failure and are consequently likely to present aging properties. Multivariate subordinators hence are good candidate to model multivariate deterioration. All the paper is written in the bivariate setting but might easily be translated into the multivariate one.

Considering bivariate subordinators as bivariate wear processes, the objective of this paper is to illustrate their ability to optimize maintenance strategies. With such an aim, we revisit a classical block replacement policy within this new context, with the optimization of a cost function as an objective. In view of the numerical assesment of the cost function, and also of an effective use for the applications, a special construction of a bivariate subordinator is proposed, by so-called trivariate reduction. Though valid in the most general setting, this construction is here proposed in the case of Gamma marginal processes, to make it clearer.

The paper is organized as follows: in Section 2, known and easy facts are summed up for univariate and bivariate subordinators. The construction of a bivariate subordinator by trivariate reduction is given in Section 3, in the case of Gamma marginal processes. The block replacement policy is presented in Section 4 and the results are numerically illustrated in Section 5. Conclusive and prospective remarks end this paper in Section 6. 


\section{BIVARIATE SUBORDINATORS}

A univariate subordinator is a univariate nondecreasing Lévy process, namely a process $\left(Y_{t}\right)_{t \geq 0}$ with range $\mathbb{R}_{+}$, starting from $Y_{0}=0$, which is non decreasing, right continuous with left-side limits and with stationary independent increments. Only subordinators with null drifts are here envisionned, both in the univariate and bivariate cases. The Laplace transform of a univariate subordinator $\left(Y_{t}\right)$ is given by:

$$
\begin{aligned}
\mathcal{L}_{Y_{t}}(x) & =\mathbb{E}\left(e^{-x Y_{t}}\right) \\
& =\exp \left(t \int_{\mathbb{R}_{+}}\left(e^{-x y}-1\right) \mu_{Y}(d y)\right)
\end{aligned}
$$

for all $x, t \geq 0$, where $\mu_{Y}(d y)$ is a non-negative measure on $\mathbb{R}_{+}^{*}$ such that

$$
\int_{\mathbb{R}_{+}}(|y| \wedge 1) \mu_{Y}(d y)<\infty
$$

where symbol $\wedge$ stands for minimum.

This measure $\mu_{Y}(d y)$ is the so-called Lévy measure of the process $\left(Y_{t}\right)_{t>0}$. The Laplace transform and the distribution of the process are characterized by its Lévy measure $\mu_{Y}(d y)$. An alternate characterization is provided by the tail integral function $U_{Y}$, with

$U_{Y}(y)=\int_{[y,+\infty[} \mu_{Y}(d x)$

for $y \in \mathbb{R}_{+}^{*}, U_{Y}(0)=+\infty$ and $U_{Y}(+\infty)=0$.

A well-known example of a univariate subordinator is the Gamma process. Its Lévy measure is equal to $\mu_{Y}(d y)=\frac{\alpha}{y} \exp (-\beta y) d y$, where $\alpha, \beta>0$. A great interest of the Gamma process is the explicit knowledge of the probability distribution of $Y_{t}$ (Gamma distributions). For other subordinators, a close form is generally not available. Nevertheless, recent researches in statistics provide tools for estimating the Lévy measure of general (univariate) subordinators, see (Comte and Genon-Catalot 2010) e.g..

Just as in the univariate case, a bivariate subordinator is a bivariate non-decreasing Lévy process (here with null drift), namely a process $\left(X_{t}=\left(X_{t}^{(1)}, X_{t}^{(2)}\right)\right)_{t \geq 0}$ with range $\mathbb{R}_{+}^{2}$, starting from $X_{0}=(0,0)$, which is component-wise nondecreasing, right continuous with left-side limits and with stationary independent increments. To avoid trivialities, $\mathbb{P}\left(X_{t}^{(1)}>0, X_{t}^{(2)}>0\right)$ is assumed to be non zero.
The Laplace transform of $X_{t}$ is

$$
\begin{aligned}
& \mathcal{L}_{X_{t}}\left(x_{1}, x_{2}\right) \\
& =\mathbb{E}\left(e^{-\left(x_{1} X_{t}^{(1)}+x_{2} X_{t}^{(2)}\right)}\right) \\
& =\exp \left(t \iint_{\mathbb{R}_{+}^{2}}\left(e^{-\left(x_{1} y_{1}+x_{2} y_{2}\right)}-1\right) \mu_{X}\left(d y_{1}, d y_{2}\right)\right)
\end{aligned}
$$

for all $\mathbf{x}=\left(x_{1}, x_{2}\right) \in \mathbb{R}_{+}^{2}$ and all $t \geq 0$.

Here again the Laplace transform and the distribution of the process are characterized by the Lévy measure $\mu_{X}$ which fulfills:

$\iint_{\mathbb{R}_{+}^{2}}(\|\mathbf{y}\| \wedge 1) \mu_{X}(d \mathbf{y})<\infty$,

where $\|\ldots\|$ stands for any standard norm on $\mathbb{R}_{+}^{2}$.

The Lévy measure can be characterized by the bivariate tail integral function $U_{X}:[0,+\infty]^{2} \rightarrow[0,+\infty]$ provided by:

$U_{X}\left(x_{1}, x_{2}\right)=\int_{\left[x_{1},+\infty[\right.} \int_{\left[x_{2},+\infty[\right.} \mu_{X}(d \mathbf{y})$

for all $\left(x_{1}, x_{2}\right) \in \mathbb{R}_{+}^{2} \backslash\{(0,0)\}, \quad U_{X}\left(x_{1}, \infty\right)=$ $U_{X}\left(\infty, x_{2}\right)=0$ and $U_{X}(0,0)=+\infty$.

The bivariate tail integral function measure the dependence between the two marginal processes in the sense that if $X$ and $\tilde{X}$ are bivariate subordinators with the same marginal processes and respective tail integral functions $U_{X}(x)$ and $U_{\tilde{X}}(x)$ such that $U_{X}(x) \leq$ $U_{\tilde{X}}(x)$ for all $x$ in $\mathbb{R}_{+}^{2}$, then the components $X_{t}^{(1)}$ and $X_{t}^{(2)}$ are "more dependent" than the components $\tilde{X}_{t}^{(1)}$ and $\tilde{X}_{t}^{(2)}$ (with clear notations). As an illustration, it is easy to check that

$U_{X}\left(x_{1}, x_{2}\right) \leq U_{X \|}\left(x_{1}, x_{2}\right)$

for all $\left(x_{1}, x_{2}\right) \in[0,+\infty]^{2}$, where

$U_{X \|}\left(x_{1}, x_{2}\right)=U_{1}\left(x_{1}\right) \wedge U_{2}\left(x_{2}\right)$

stands for the tail integral function in the case where the components are completely dependent (case of maximal dependence). The maximal dependence is hence reached for the maximal tail integral function.

Given a bivariate subordinator $\left(X_{t}^{(1)}, X_{t}^{(2)}\right)_{t \geq 0}$, for each $i=1,2$, the marginal process $\left(X_{t}^{(i)}\right)_{t \geq 0}$ is a univariate subordinator with associated Lévy measure $\mu_{i}$ where $\mu_{1}\left(d x_{1}\right)=\mu_{X}\left(d x_{1} \times \mathbb{R}_{+}\right)$(the same for $\mu_{2}$ ) and (univariate) tail integral function denoted by $U_{i}$. 
The linear correlation coefficient $\rho_{X_{t}}$ of $X_{t}$ is a measure of the dependence between $X_{t}^{(1)}$ and $X_{t}^{(2)}$. Under technical assumptions ensuring its existence, a direct calculation of covariance and standard deviations with the help of Laplace transforms (1) and (2) gives:

$\rho_{X_{t}}=\frac{\iint_{\mathbb{R}_{+}^{2}} U_{X}\left(u_{1}, u_{2}\right) d u_{1} d u_{2}}{\sqrt{v_{1} v_{2}}}$

with

$v_{i}=\operatorname{var}\left(X_{1}^{(i)}\right)=2 \int_{\mathbb{R}_{+}} u U_{i}(u) d u$

for $i=1,2$. Expression (5) shows that $\rho_{X_{t}}$ is independent of $t$ (just as for any Lévy process). It is denoted by $\rho_{X}$ in the following.

Using $0 \leq U_{X}\left(x_{1}, x_{2}\right)$ and (4), we derive the following bounds for $\rho_{X}$ :

$0 \leq \rho_{X} \leq \rho_{\max }$

with

$\rho_{\max }:=\frac{\iint_{\mathbb{R}_{+}^{2}}\left(U_{1}\left(u_{1}\right) \wedge U_{2}\left(u_{2}\right)\right) d u_{1} d u_{2}}{\sqrt{v_{1} v_{2}}}$

The lower bound $\rho_{X}=0$ corresponds to the case of independent indicators. The upper bound $\rho_{\max }$ is reachable and corresponds to the case of complete (maximal) dependence. We have $\rho_{\max }=1$ in case $U_{1}=U_{2}$, but in general $\rho_{\max }<1$, see Figure 1 later on. This result shows that, in case of non equal marginal subordinators, high dependence between the marginal processes cannot always be modeled by a bivariate subordinator.

We finally recall a comparison result from (Bäuerle et al. 2008), to be used later on. This result roughly says that a bivariate subordinator is "increasing" with dependence. More specifically, if $X$ and $\tilde{X}$ are bivariate subordinators with the same marginal processes and respective tail integral functions $U_{X}(x)$ and $U_{\tilde{X}}(x)$ such that $U_{X}(x) \leq U_{\tilde{X}}(x)$ for all $x$ in $\mathbb{R}_{+}^{2}$, then $X$ is smaller than $\tilde{X}$ in the sense of the concordance order. This means that:

$\mathbb{P}\left(X_{t}^{(1)}>s_{1}, X_{t}^{(2)}>s_{2}\right) \leq \mathbb{P}\left(\tilde{X}_{t}^{(1)}>s_{1}, \tilde{X}_{t}^{(2)}>s_{2}\right)$,

$\mathbb{P}\left(X_{t}^{(1)} \leq s_{1}, X_{t}^{(2)} \leq s_{2}\right) \leq \mathbb{P}\left(\tilde{X}_{t}^{(1)} \leq s_{1}, \tilde{X}_{t}^{(2)} \leq s_{2}\right)$,

for all $t, s_{1}, s_{2} \geq 0$.

As already told, there is a special interest for bivariate subordinators with Gamma marginal processes, because the marginal probability distributions are explicitly known (Gamma distributions). However, no closed form is generally available for the process bivariate distribution. Nevertheless the construction given in the next section provides a special case where such a distribution can be obtained.

\section{CONSTRUCTION BY TRIVARIATE REDUCTION}

We here propose a model of bivariate subordinators constructed by trivariate reduction (TR subordinator), in the special case of Gamma marginal processes. This method has already been introduced in (Buijs et al. 2005) or Ebrahimi, and used in (Mercier et al. 2009). Results on bivariate Gamma distributions constructed by trivariate reduction may also be found in Devroye (Devroye 1986, section XI.3).

Let us first recall that the probability distribution function (p.d.f.) of a Gamma distribution $\Gamma(a, b)$ is

$f_{a, b}(u)=\frac{b^{a}}{\Gamma(a)} u^{a-1} e^{-b u} \mathbf{1}_{\mathbb{R}_{+}}(u)$

and that the distribution at time $t$ of a univariate Gamma process with parameters $(a, b)$ is $\Gamma(a t, b)$. The tail integral function of a Gamma process with parameters $(a, b)$ is:

$U(x)=\int_{x}^{+\infty} a \frac{e^{-b u}}{u} d u=a E_{1}(b x)$

where $E_{1}(x)=\int_{x}^{+\infty} \frac{e^{-y}}{y} d y$ is the exponential integral function.

For a general subordinator with univariate Gamma processes as margins and marginal parameters $\left(a_{1}, b_{1}\right)$ and $\left(a_{2}, b_{2}\right)$, the upper bound $(6)$ is then provided by:

$\rho_{\text {max }}(\alpha)$

$=\iint_{\mathbb{R}_{+}^{2}} \min \left(\sqrt{\alpha} E_{1}\left(u_{1}\right), \frac{1}{\sqrt{\alpha}} E_{1}\left(u_{2}\right)\right) d u_{1} d u_{2}$

where $\alpha=\frac{a_{1}}{a_{2}}$.

Let us now describe the trivariate construction: starting from three independent univariate Gamma processes $\left(Y_{t}^{(i)}\right)_{t \geq 0}$ with parameters $\left(\alpha_{i}, 1\right)$ for $i \in$ $\{1,2,3\}$ and from $\bar{b}_{1}, b_{2}>0$, we set:

$\left\{\begin{array}{l}X_{t}^{(1)}=\left(Y_{t}^{(1)}+Y_{t}^{(3)}\right) / b_{1} \\ X_{t}^{(2)}=\left(Y_{t}^{(2)}+Y_{t}^{(3)}\right) / b_{2}\end{array}\right.$

The interest of such a construction is clear for the applications to reliability, where the term $Y_{t}^{(3)}$ will correspond to some common damage accumulation coming from some common wear source, whereas $Y_{t}^{(1)}$ and $Y_{t}^{(2)}$ will correspond to some damage accumulations coming from independent wear sources.

The process $\left(X_{t}\right)_{t \geq 0}=\left(X_{t}^{(1)}, X_{t}^{(2)}\right)_{t \geq 0}$ constructed in (9) is a bivariate subordinator with univariate Gamma processes as margins. Its marginal parameters $\left(a_{i}, b_{i}\right)$ are provided by:

$\left\{\begin{array}{l}a_{1}=\alpha_{1}+\alpha_{3} \\ a_{2}=\alpha_{2}+\alpha_{3}\end{array}\right.$ 


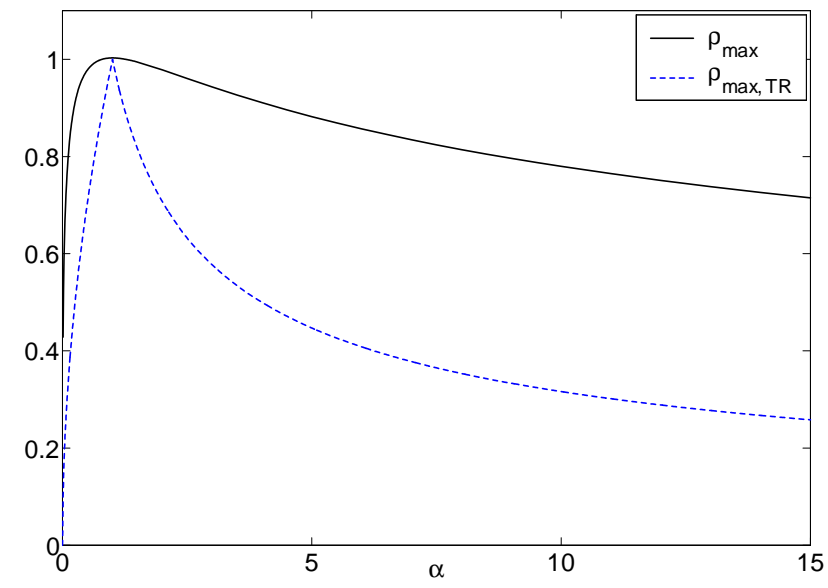

Figure 1: $\rho_{\max }(\alpha)$ and $\rho_{\max }, T R(\alpha)$ with respect of $\alpha$.

A direct computation provides:

$\rho_{X}=\frac{\alpha_{3}}{\sqrt{a_{1} a_{2}}}$

and setting $\rho=\rho_{X}$ :

$\left\{\begin{array}{c}\alpha_{1}=a_{1}-\rho \sqrt{a_{1} a_{2}} \\ \alpha_{2}=a_{2}-\rho \sqrt{a_{1} a_{2}} \\ \alpha_{3}=\rho \sqrt{a_{1} a_{2}}\end{array}\right.$

Two equivalent alternate parametrizations hence are available for TR subordinators with Gamma marginal processes: either $\left(\alpha_{1}, \alpha_{2}, \alpha_{3}, b_{1}, b_{2}\right)$ or $\left(a_{1}, a_{2}, \rho, b_{1}, b_{2}\right)$. This entails that all the dependence of a TR subordinator with Gamma marginal processes is contained in the linear correlation coefficient $\rho$. Equations (10) also entail that:

$\rho \leq \frac{\min \left(a_{1}, a_{2}\right)}{\sqrt{a_{1} a_{2}}}=\min \left(\sqrt{\alpha}, \frac{1}{\sqrt{\alpha}}\right):=\rho_{\max , T R}(\alpha)$

where $\alpha:=\frac{a_{1}}{a_{2}}$.

In case $a_{1}=a_{2}$, we get $\rho_{\max , T R}=\rho_{\max }=1$. In the general case, we however get $\rho_{\max }, T R<\rho_{\max }$, as shows Figure 1 where the functions $\alpha \longmapsto \rho_{\max }(\alpha)$ and $\alpha \longmapsto \rho_{\max , T R}(\alpha)$ are plotted. This proves that a trivariate construction cannot model as high a dependence as general subordinators, in case of different marginal processes.

For the practical use of TR subordinators in Section 5 , we now provide the joint p.d.f. $f_{X_{t}}\left(u_{1}, u_{2}\right)$ of $X_{t}$ : when $\alpha_{3} \neq 0$, it is equal to:

$$
\int_{0}^{\min \left(u_{1}, u_{2}\right)} f_{\alpha_{1} t, 1}\left(u_{1}-u_{3}\right) f_{\alpha_{2} t, 1}\left(u_{2}-u_{3}\right) f_{\alpha_{3} t, 1}\left(u_{3}\right) d u_{3}
$$

When $\alpha_{3}=0$ (or $\rho=0$ ), both $X_{t}^{(1)}$ and $X_{t}^{(2)}$ are independent and $f_{X_{t}}\left(u_{1}, u_{2}\right)=f_{a_{1} t}\left(u_{1}\right) f_{a_{2} t}\left(u_{2}\right)$.

\section{BLOCK REPLACEMENT POLICY}

In this section we illustrate the ability of using bivariate subordinator to optimize maintenance strategy with a block replacement policy: a system is considered, with degradation measured by a bivariate subordinator $\left(X_{t}\right)_{t \geq 0}$ (with null drift). For each univariate subordinator $\left(X_{t}^{(i)}\right)_{t \geq 0}$, there is a threshold $s_{i}$ above which the deterioration level is considered as too high and we set:

$\sigma_{s_{i}}^{(i)}=\inf \left(t \geq 0\right.$ such that $\left.X_{t}^{(i)} \geq s_{i}\right)$

to be the corresponding univariate reaching time $(i=$ $1,2)$. The random variable $\sigma^{(i)}$ hence stands for a univariate time to failure, only taking indicator $X_{t}^{(i)}$ into consideration. As for the bivariate case, we envision two possibilities for the system failure: either the system is failed as soon as one (univariate) indicator is too high or when both indicators are. If we interpret $X_{t}^{(1)}$ and $X_{t}^{(2)}$ as the wear indicators of two dependent components, the first case corresponds to a seriessystem and the second one to a parallel system. The respective times to failure are:

$$
\begin{aligned}
\sigma & =\inf \left(t \geq 0 \mid X_{t}^{(1)} \geq s_{1} \text { or } X_{t}^{(2)} \geq s_{2}\right) \\
& =\min \left(\sigma_{s_{1}}^{(1)}, \sigma_{s_{2}}^{(2)}\right)
\end{aligned}
$$

and

$$
\begin{aligned}
\sigma & =\inf \left(t \geq 0 \mid X_{t}^{(1)} \geq s_{1} \text { and } X_{t}^{(2)} \geq s_{2}\right) \\
& =\max \left(\sigma_{s_{1}}^{(1)}, \sigma_{s_{2}}^{(2)}\right) .
\end{aligned}
$$

The block replacement policy is then applied: the system is not continuously observed. It is perfectly and instantaneously repaired each $T$ time unit (periodically) with a cost $c_{r}$. If the system is down at repair time, a unitary cost $c_{u}$ is induced per unit time for the down period just before the repair. After a repair, the future evolution of the system is similar as from the beginning and is independent of its past. We are interested in the asymptotic unitary cost (per unit time) denoted by $C(T)$. We first recall classical results for sake of completeness, see (Nakagawa 2005) e.g..

Using standard renewal theory, the asymptotic unitary cost $C(T)$ is provided by:

$C(T)=\frac{\mathbb{E}(C([0, T]))}{T}$

where $\mathbb{E}(C([0, T]))$ is the mean cost on a generic cycle $[0, T]$.

The asymptotic unitary cost then is:

$C(T)=\frac{c_{r}+c_{u} \int_{0}^{T} F_{\sigma}(t) d t}{T}$ 
where $F_{\sigma}(t)$ is the cumulative distribution function (c.d.f.) of the failure time $\sigma$.

A finite optimal $T^{o p t}$ exists if and only if $\mathbb{E}(\sigma)>\frac{c_{r}}{c_{u}}$. In that case, this optimal point is characterized by

$$
\int_{0}^{T^{o p t}} t f_{\sigma}(t) d t=\frac{c_{r}}{c_{u}}
$$

and the optimal cost is:

$C\left(T^{o p t}\right)=c_{u} F_{\sigma}\left(T^{o p t}\right)$.

In case, $\mathbb{E}(\sigma) \leq \frac{c_{r}}{c_{u}}$, the cost function $C(T)$ is non increasing with $T$ and the best is not to ever repair the system $\left(T^{o p t}=+\infty\right)$.

We now complete such results in the present context and we first consider the case where $\sigma=$ $\min \left(\sigma_{s_{1}}^{(1)}, \sigma_{s_{2}}^{(2)}\right)$.

In that case, we have:

$$
\begin{aligned}
F_{\sigma}(t) & =\mathbb{P}(\sigma \leq t) \\
& =1-\mathbb{P}\left(X_{t}^{(1)} \leq s_{1}, X_{t}^{(2)} \leq s_{2}\right)
\end{aligned}
$$

for all $t \geq 0$ and

$$
\begin{aligned}
\mathbb{E}(\sigma) & =\int_{0}^{+\infty} \mathbb{P}(\sigma>t) d t \\
& =\int_{0}^{+\infty} \mathbb{P}\left(X_{t}^{(1)} \leq s_{1}, X_{t}^{(2)} \leq s_{2}\right) d t
\end{aligned}
$$

As a consequence, it can be seen from $(7)$ that $F_{\sigma}(t)$, $C(T)$ and $C\left(T^{o p t}\right)$ are non increasing with dependence (see (13) and (14)), whereas $\mathbb{E}(\sigma)$ is non decreasing with dependence. This entails that

$\mathbb{E}\left(\sigma^{\perp}\right) \leq \mathbb{E}(\sigma) \leq \mathbb{E}\left(\sigma^{\|}\right)$

where $\mathbb{E}\left(\sigma^{\perp}\right)$ and $\mathbb{E}\left(\sigma^{\|}\right)$stand for the mean times to failure in case of independent and completely dependent wear indicators, respectively (with fixed margins).

In case $\mathbb{E}\left(\sigma^{\perp}\right)>\frac{c_{r}}{c_{u}}$, we hence have $\mathbb{E}(\sigma)>\frac{c_{r}}{c_{u}}$ and a finite $T^{o p t}$ exists for any kind of dependence.

If $\mathbb{E}\left(\sigma^{\|}\right) \leq \frac{c_{r}}{c_{u}}$, a finite $T^{o p t}$ never exists.

If $\mathbb{E}\left(\sigma^{\perp}\right) \leq \frac{c_{r}}{c_{u}}<\mathbb{E}\left(\sigma^{\|}\right)$, a finite $T^{o p t}$ exists for high dependence but does not exist for low dependence.

This shows the clear influence of the dependence on the optimal maintenance policy.

Similar results are valid in case $\sigma=$ $\max \left(\sigma_{s_{1}}^{(1)}, \sigma_{s_{2}}^{(2)}\right)$. In that case, we get:

$$
\begin{aligned}
& F_{\sigma}(t)=\mathbb{P}\left(X_{t}^{(1)}>s_{1}, X_{t}^{(2)}>s_{2}\right) \\
& \mathbb{E}(\sigma)=\int_{0}^{+\infty}\left(1-\mathbb{P}\left(X_{t}^{(1)}>s_{1}, X_{t}^{(2)}>s_{2}\right)\right) d t
\end{aligned}
$$

and all conclusions are reversed. In this way, $F_{\sigma}(t)$, $C(T)$ and $C\left(T^{o p t}\right)$ are non decreasing with dependence, whereas $\mathbb{E}(\sigma)$ is non increasing with dependence. In case $\mathbb{E}\left(\sigma^{\|}\right) \leq \frac{c_{r}}{c_{u}}<\mathbb{E}\left(\sigma^{\perp}\right)$, a finite $T^{o p t}$ exists for low dependence but does not exist for high dependence.

\section{NUMERICAL EXPERIMENTS}

This section illustrates the previous results for a bivariate subordinator with Gamma marginal processes, constructed by trivariate reduction (see Section 3 ).

\subsection{Numerical computations}

Based on the explicit form (12) of the p.d.f. of $\left(X_{t}^{(1)}, X_{t}^{(2)}\right)$, it is possible to compute all the quantities analytically provided in Section 4.

As an example, in case $\sigma=\min \left(\sigma_{s_{1}}^{(1)}, \sigma_{s_{2}}^{(2)}\right)$ and $\rho>0$, we have:

$$
\begin{aligned}
F_{\sigma}(t)=1-\int_{0}^{s_{1}} \int_{0}^{s_{2}} f_{X_{t}}\left(u_{1}, u_{2}\right) d u_{1} d u_{2} \\
=1-\int_{0}^{\min \left(s_{1}, s_{2}\right)} F_{\alpha_{1} t, 1}\left(s_{1}-u_{3}\right) \\
\quad \times F_{\alpha_{2} t, 1}\left(s_{2}-u_{3}\right) f_{\alpha_{3} t, 1}\left(u_{3}\right) d u_{3}
\end{aligned}
$$

where $F_{a, b}$ is the cumulative distributive function of the Gamma distribution with parameters $a$ and $b$.

This provides:

$$
\int_{0}^{T} F_{\sigma}(t) d t
$$

$$
\begin{gathered}
=T-\int_{0}^{T} \int_{0}^{\min \left(s_{1}, s_{2}\right)} F_{\alpha_{1} t, 1}\left(s_{1}-u_{3}\right) F_{\alpha_{2} t, 1}\left(s_{2}-u_{3}\right) \\
\times f_{\alpha_{3} t, 1}\left(u_{3}\right) d u_{3} d t
\end{gathered}
$$

Due to numerical difficulties, we actually prefer 
write:

$$
\begin{aligned}
& \int_{0}^{T} F_{\sigma}(t) d t \\
& =T-\int_{0}^{T} \int_{0}^{\min \left(s_{1}, s_{2}\right)}\left(1-\bar{F}_{\alpha_{1} t, 1}\left(s_{1}-u_{3}\right)\right) \\
& \quad \times\left(1-\bar{F}_{\alpha_{2} t, 1}\left(s_{2}-u_{3}\right)\right) f_{\alpha_{3} t, 1}\left(u_{3}\right) d u_{3} d t \\
& =\int_{0}^{T} \bar{F}_{\alpha_{3} t, 1}\left(\min \left(s_{1}, s_{2}\right)\right) d t \\
& +\int_{0}^{T} \int_{0}^{\min \left(s_{1}, s_{2}\right)} \bar{F}_{\alpha_{1} t, 1}\left(s_{1}-u_{3}\right) f_{\alpha_{3} t, 1}\left(u_{3}\right) d u_{3} d t \\
& +\int_{0}^{T} \int_{0}^{\min \left(s_{1}, s_{2}\right)} \bar{F}_{\alpha_{2} t, 1}\left(s_{2}-u_{3}\right) f_{\alpha_{3} t, 1}\left(u_{3}\right) d u_{3} d t \\
& -\int_{0}^{T} \int_{0}^{\min \left(s_{1}, s_{2}\right)} \bar{F}_{\alpha_{1} t, 1}\left(s_{1}-u_{3}\right) \bar{F}_{\alpha_{2} t, 1}\left(s_{2}-u_{3}\right) \\
& \times f_{\alpha_{3} t, 1}\left(u_{3}\right) d u_{3} d t
\end{aligned}
$$

and compute the four involved quantities, which provides more accurate results.

When $\rho=0$, the components are independent and, in case $\sigma=\min \left(\sigma_{s_{1}}^{(1)}, \sigma_{s_{2}}^{(2)}\right)$, we get:

$$
F_{\sigma}(t)=1-F_{\alpha_{1} t, 1}\left(s_{1}\right) F_{\alpha_{2} t, 1}\left(s_{2}\right) \text {. }
$$

Different numerical experiments are next presented in order to illustrate the behaviour of $C(T), T^{o p t}$ and $C\left(T^{o p t}\right)$ with respect of $T$ and/or $\rho$. As for the influence of the other parameters, $C(T)$ is clearly increasing with respect of $c_{r}, c_{u}, a_{1}, a_{2}, b_{1}$ and $b_{2}$, and decreasing with respect of $s_{1}$ and $s_{2}$, so that no further study is required.

Examples 1 to 3 correspond to the case where $\sigma=$ $\min \left(\sigma_{s_{1}}^{(1)}, \sigma_{s_{2}}^{(2)}\right)$.

\subsection{Example 1}

The parameters of the process are $a_{1}=50, a_{2}=70$, $b_{1}=b_{2}=1, \rho=0.7$. The failure levels are $s_{1}=50$ and $s_{2}=40$. The costs are $c_{r}=1$ and $c_{u}=50$. Figure 2 gives $C(T)$ with respect to $T$ and the asymptotic unitary costs $C^{(i)}(T)$ for each univariate indicator $(i=1,2)$. Such unitary costs are computed taking into account one single component $X_{t}^{(i)}$, which means that the time to failure is considered to be $\sigma^{(i)}$. The induced costs are assumed to be the same as for the bivariate case. Both components are renewed at the same time when the system is renewed and this induces the same renewal cost for the bivariate and univariate cases. In Figure 2, one can observe that $C(T)$ and $C^{(2)}(T)$ are nearly superimposed and are clearly higher than $C^{(1)}(T)$. This comes from the fact that the different parameters are such that $\mathbb{E}\left(\sigma_{s_{1}}^{(1)}\right) \simeq 1.01$ and $\mathbb{E}\left(\sigma_{s_{2}}^{(2)}\right) \simeq 0.58$. As a consequence, the second component $X_{t}^{(2)}$ will fail before the first one $X_{t}^{(1)}$ with a high probability and in that case, it is useless to use both components $X_{t}^{(1)}$ and $X_{t}^{(2)}$. In case of very different components, the bivariate model consequently does not bring much and one can simply consider the "worse" one.

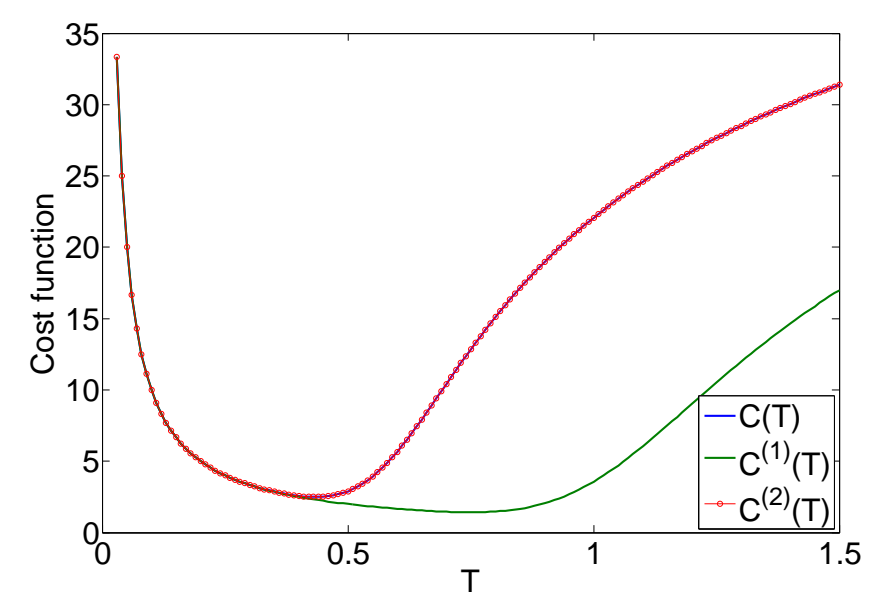

Figure 2: $C(T)$ with respect of $T$.

\subsection{Example 2}

The parameters of the marginal processes are $a_{1}=50$, $a_{2}=74, b_{1}=b_{2}=1$. The failure levels are $s_{1}=20$ and $s_{2}=30$. The costs are $c_{r}=10$ and $c_{u}=500$. In this case $a_{1}<a_{2}, s_{1}<s_{2}$ but the mean univariate times to failures are close: $\mathbb{E}\left(\sigma_{s_{1}}^{(1)}\right) \simeq \mathbb{E}\left(\sigma_{s_{2}}^{(2)}\right) \simeq$ 0.41 . The function $C(T)$ is computed for $\rho=0$, and $\rho=\rho_{\max }^{T R}$. The results are presented in Figure 3, as well as $C^{(i)}(T)$ for $i=1,2$. In that case, the univariate costs $C^{(i)}(T)$ are very close one from each other. The bivariate cost $C(T)$ is observed to be higher. In that case, not taking into account both components may lead to an under-estimated cost fonction.

\subsection{Example 3}

The parameters of the marginal processes are $a_{1}=$ $a_{2}=50, b_{1}=b_{2}=1$. The failure levels are $s_{1}=s_{2}=$ 30 . The costs are $c_{r}=10$ and $c_{u}=500$. The two components have the same distribution and the same failure level, so that the marginal times to failure are identical. The cost function $C(T)$ is computed for different values of $\rho$ and the results are presented in Figure 4. As expected, $C(T)$ is observed to be decreasing with dependence. In case of an unknown correlation, 


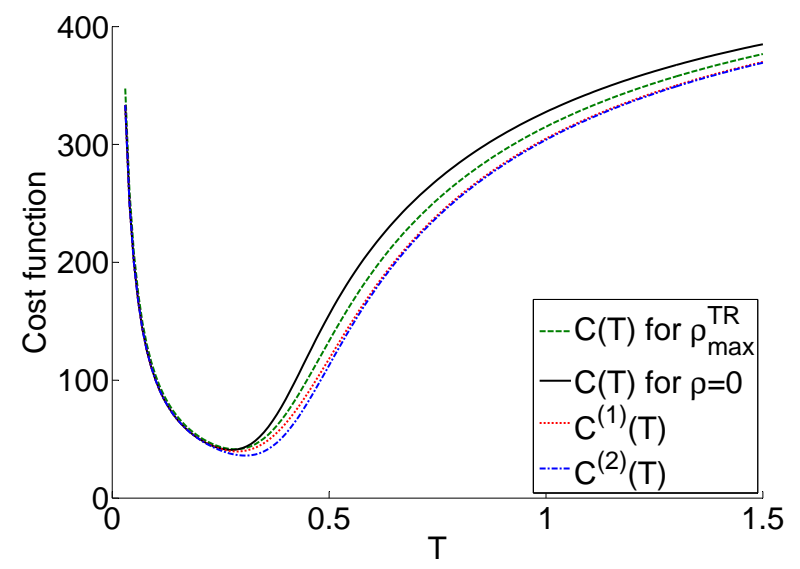

Figure 3: $C(T)$ with respect of $T$ for $\rho=\rho_{\max }^{T R}, \rho=0$ and $C^{(i)}(T)$ for $i=1,2$.

the most conservative attitude hence is to take into account both components and to consider them as independent. Also, the univariate costs $C^{(i)}(T)$ for $i=1,2$ coïncides with the bivariate cost function $C(T)$ in case $\rho=1$. Taking into consideration one single indicator hence lead to an under-estimated cost fonction.

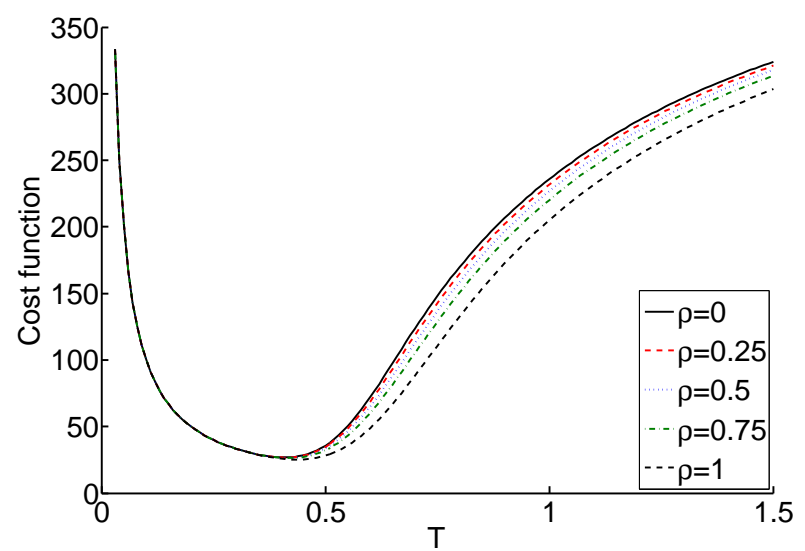

Figure 4: $C(T)$ with resp. of $T$ for $\rho=0,0.25,0.5,0.75$ and 1.

The optimal cost $C\left(T^{o p t}\right)$ and period $T^{o p t}$ are next plotted against the linear correlation coefficient $\rho$ in Figures 5 and 6. As expected, $C\left(T^{o p t}\right)$ is nonincreasing with dependence. The optimal period $T^{o p t}$ is observed to be non-decreasing with dependence. That point has not been proved from a theoretical point of view.

\subsection{Example 4}

The numerical parameters are the same as for example 3 , but the time to failure now is $\sigma=\max \left(\sigma_{s_{1}}^{(1)}, \sigma_{s_{2}}^{(2)}\right)$. The results are provided in Figures 7 and 8. As expected, the monotony of $C\left(T^{o p t}\right)$ and $T^{o p t}$ with dependence is reversed. The optimal cost is here a little lower than in case $\sigma=\min \left(\sigma_{s_{1}}^{(1)}, \sigma_{s_{2}}^{(2)}\right)$, and the optimal period a little higher. This is conform to what is

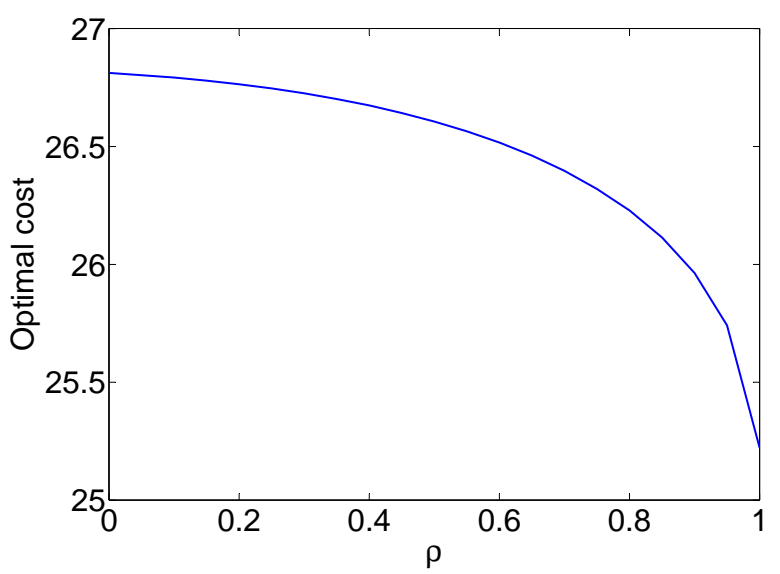

Figure 5: $C^{o p t}(\rho)$ with respect of $\rho$.

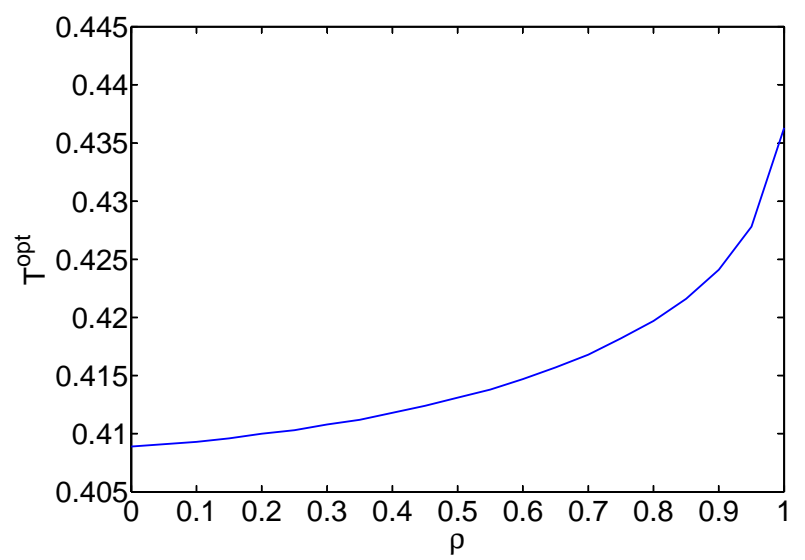

Figure 6: $T^{o p t}(\rho)$ with respect of $\rho$.

expected, because a parallel-system is more reliable than a series-system.

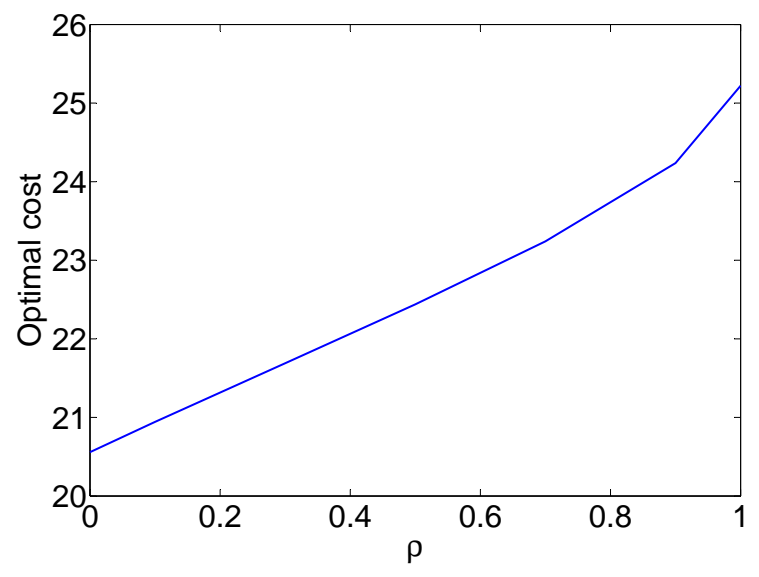

Figure 7: $C^{o p t}(\rho)$ with respect of $\rho$.

\subsection{Conclusion, Prospective remarks}

A block replacement policy has been studied in the new context of a bivariate deterioration modelled by a bivariate subordinator. The influence of the dependence between the marginal wear indicators on the optimal policy has been pointed out. In the case where 


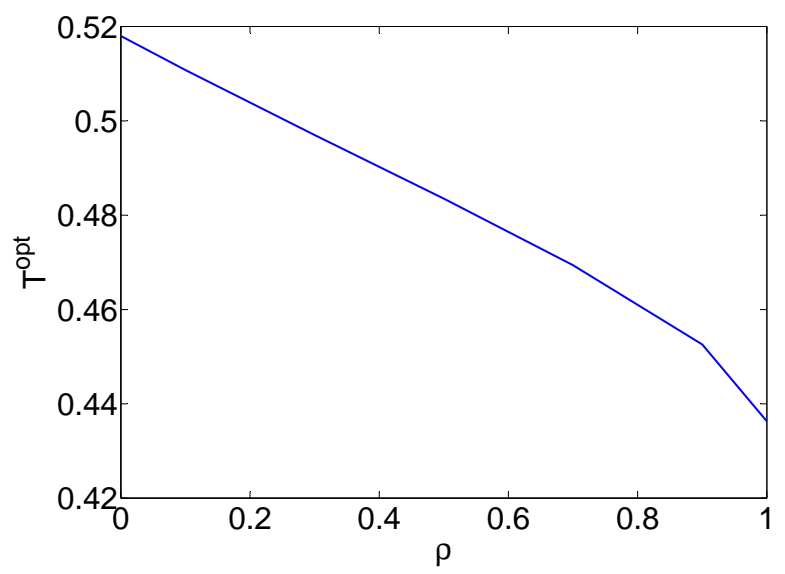

Figure 8: $T^{o p t}(\rho)$ with respect of $\rho$.

the system time to failure is the minimum or the maximum of the two univariate times to failure, the optimal cost function has been proved to be monotone with respect to the dependence, with opposite monotonicities for the two cases. When the correlation is unknown, the safest attitude hence is to consider the correlation which leads to the highest cost, namely consider the components as independent in the first case (minimum) and completely dependent in the second case (maximum).

As for the optimal period, it has been numerically observed to be monotone with dependence for all the examples provided here, as well as for all the other examples we may have looked at. It may then be conjectured that this property is always true in the studied context. However, we have not been able to prove it theoretically. This consequently remains on open question.

In the present paper, the choice has been made to consider a simplified model where the system time to failure is the minimum or the maximum of the two univariate times to failure. Thinking of the two components $X_{t}^{(1)}$ and $X_{t}^{(2)}$ as the width and heigth of a girder for instance, one may think that a more adequate criterion for high deterioration might be linked to the grid section $X_{t}^{(1)}$. $X_{t}^{(2)}$ e.g.. The time to failure might then has the following shape:

$$
\begin{aligned}
\sigma & =\inf \left(t \geq 0: X_{t}^{(1)} \cdot X_{t}^{(2)} \geq s\right) \\
& =\inf \left(t \geq 0:\left(X_{t}^{(1)}, X_{t}^{(2)}\right) \in \mathcal{S}\right)
\end{aligned}
$$

where $s$ is some fixed threshold and $\mathcal{S}=\left\{\left(x_{1}, x_{2}\right) \in \mathbb{R}_{+}^{2}: x_{1} x_{2} \geq s\right\}$. More generally, failure times may correspond to reaching times of failure zones, which may be quite general $(S$ should be an upper set namely such that if $\left(x_{1}, x_{2}\right) \in S$ and $\left(x_{1}, x_{2}\right) \leq\left(y_{1}, y_{2}\right)$, then $\left.\left(y_{1}, y_{2}\right) \in \mathcal{S}\right)$. Further studies should then be performed for such general failure zones.

Also, the choice has here been made to renew both indicators at each maintenance time. In case of indi- cators linked to two different components, a wiser attitude might be to renew a component only if it is too deteriorated $\left(X_{t}^{(i)} \geq M_{i}\right.$, where $M_{i}$ is some maintenance threshold). Such a maintenance policy also requires further investigation.

\section{REFERENCES}

Abdel-Hameed, M. (1975). A gamma wear process. IEEE Trans. Reliability 24(2), 152153.

Bäuerle, N., A. Blatter, \& A. Müller (2008). Dependence properties and comparison results for lévy processes. Math. Methods Oper. Res. 67(1), 161-186.

Buijs, F. A., J. W. Hall, J. M. Van Noortwijk, \& P. B. Sayers (2005). Time dependent reliability analysis of flood defences using gamma processes. In G. Augusti, G. I. Schüeller, and M. Ciampoli (Eds.), Safety and Reliability of Engineering Systems and Structures; Proceedings of the Ninth International Conference on Structural Safety and Reliability (ICOSSAR), Rome, Italy, 19-23 June 2005, Rotterdam, pp. 2209-2216. Millpress.

Comte, F. \& V. Genon-Catalot (2010). Nonparametric adaptive estimation for pure jump Lévy processes. Ann. Inst. H. Poincar Probab. Statist. 46(3), 595-617.

Devroye, L. (1986). Non-uniform random variate generation. New York: Springer-Verlag.

Mercier, S., C. Meier-Hirmer, \& M. Roussignol (2009). Modelling track geometry by a bivariate gamma wear process, with application to maintenance. In M. Kallen and S. P. Kuniewski (Eds.), Risk and Decision Analysis in Maintenance Optimization and Flood Management. IOS Press, Delft.

Mercier, S., C. Meier-Hirmer, \& M. Roussignol (2011). Bivariate Gamma wear processes for track geometry modelling, with application to intervention scheduling. Structure and Infrastructure Engineering. Published Online.

Nakagawa, T. (2005). Block replacement. In Maintenance Theory of Reliability, Springer Series in Reliability Engineering, pp. 117-134. Springer London.

Nakagawa, T. (2007). Shock and damage models in reliability theory. Springer-Verlag New York Inc.

Van Noortwijk, J. (2009). A survey of the application of Gamma processes in maintenance. $R e$ liab. Eng. Syst. Saf. 94(1), 2-21. 